\title{
Gas disks and supermassive black holes in nearby radio galaxies
}

\author{
Jacob Noel-Storr ${ }^{1}$, Stefi Baum ${ }^{2}$, Chris O'Dea ${ }^{2}$ \\ and Gijs Verdoes Kleijn ${ }^{3}$ \\ ${ }^{1}$ Department of Astronomy, Columbia University, New York, NY 10027, USA \\ email: jake@astro.columbia.edu \\ ${ }^{2}$ Space Telescope Science Institute, USA \\ ${ }^{3}$ European Southern Observatory, Germany
}

\begin{abstract}
We present the initial results of our analysis of line emission produced in gas disks found at the centers of a sample of nearby radio galaxies with radio jets. We obtained data using STIS (The Space Telescope Imaging Spectrograph) at three parallel slit positions on the nucleus of each galaxy. This allows us to map the $\mathrm{H} \alpha+[\mathrm{NII}]$ flux, the gas radial velocity and the velocity dispersion. We found that we cannot rule out a rotating disk model for the gas in any case. We found that for $62 \%$ of the galaxies the fit is improved in the nucleus by the inclusion of an additional broad component. By comparing the observed central kinematics to model gas disks we were able to estimate the masses of nuclear black holes in 12 of the sample galaxies.
\end{abstract}

The UGC FR-I sample is a complete sample of 21 northern hemisphere radio galaxies with jets (see Noel-Storr et al. 2003; Xu et al. 2000; Verdoes Kleijn et al. 1999); all of the galaxies are classified as FR-Is (Fanaroff \& Riley 1974). We obtained long slit spectra of each sample member using the Space Telescope Imaging Spectrograph (STIS) on board the Hubble Space Telescope (HST). We employed the G750M grating (see Brown et al. 2002 ), centered in the region of $\mathrm{H} \alpha,[\mathrm{NII}]$ and [SII], and a slit width of $0.1^{\prime \prime}$ or $0.2^{\prime \prime}$. We fit each spectral row using a flat continuum level, and five Gaussians to represent the spectral lines. Full details of the observations, data reduction and line fitting, including the full data set, are given in Noel-Storr et al. (2003).

We found that for $62 \%$ of the nuclei the fit is improved by the inclusion of an additional broad component. The detection of this broad feature is flux limited and does not relate to the dust morphology or inclination. Mean properties of the broad lines are compared with other samples in Table 1. The broad components may originate as a consequence of asymmetrical line shapes, or from physical broad line regions. As Barth et al. (1999) found a broad component in polarized light in the nucleus of NGC 315, we suspect that at least some fraction of these components originates in physical broad line regions (Noel-Storr 2004, Chapter 5).

While the observed velocity profiles are somewhat messy, and show signs that the gas is unsettled (Noel-Storr 2004, Chapter 5), we find signs of rotation in $67 \%$ of the galaxies, and that the kinematics of the other sample members are compatible with rotation.

For each galaxy we produced models representing the rotation of a thin gas disk in the major plane of the stellar potential, including black holes with masses $M_{\bullet}=0,1 \times 10^{8} M_{\odot}$ and $9 \times 10^{8} M_{\odot}$, chosen to be representative of the sizes of supermassive black holes anticipated in these galaxies. The calculated velocity profiles were convolved with the instrument pixel size and PSF to produce model velocity profiles that can be directly compared with the STIS observations. Details of the models are presented in Chapter 4 of Noel-Storr (2004). 


$\begin{array}{lrrr}\text { Parameter } & \text { UGC FR-I sample* }^{*} & \text { Sy/LINERs } & \text { Radio Loud }^{\ddagger} \\ <\sigma_{B L}>\left(\mathrm{km} \mathrm{s}^{-1}\right) & 1295 \pm 218 & 2400 \pm 1100 & 5900 \pm 3700 \\ <\sigma_{N L}>\left(\mathrm{km} \mathrm{s}^{-1}\right) & 371 \pm 153 & \ldots & \ldots \\ <v_{N L}-v_{B L}>\left(\mathrm{km} \mathrm{s}^{-1}\right) & -435 \pm 335 & \ldots & -500 \pm 1200\end{array}$

Table 1. Comparison of broad line statistics. We show the mean velocity dispersions of broad and narrow lines and offsets of broad and narrow lines for three different galaxy samples: *The data presented in this work; ${ }^{\dagger}$ A sample of Seyferts and LINERs (Ho et al. 1997); and ${ }^{\ddagger}$ A summary of properties of several samples of radio galaxies (Sulentic et al. 2000).

$\begin{array}{lclclc}\text { Galaxy } & M_{\bullet}\left(10^{8} M_{\odot}\right) & \text { Galaxy } & M_{\bullet}\left(10^{8} M_{\odot}\right) & \text { Galaxy } & M_{\bullet}\left(10^{8} M_{\odot}\right) \\ \text { NGC 193 } & 1<M_{\bullet}<9 & \text { NGC 383 } & M_{\bullet}>9 ? & \text { NGC 2329 } & M_{\bullet}<1^{\dagger} \\ \text { NGC 4261 } & M_{\bullet} \sim 1^{*} & \text { NGC 4335 } & M_{\bullet} \lesssim 1 & \text { M84 } & M_{\bullet}>9 \\ \text { NGC 4486 } & M_{\bullet}>9 & \text { NGC 5127 } & 1<M_{\bullet}<9 & \text { NGC 5141 } & M_{\bullet} \lesssim 1 \\ \text { NGC 7052 } & 1<M_{\bullet}<9 & \text { UGC 12064 } & M_{\bullet} \sim 9 & \text { NGC 7626 } & 1<M_{\bullet}<9\end{array}$

Table 2. Black hole masses compatible with the observed kinematic profiles in the central regions of each galaxy. ${ }^{*}$ NGC 4261 is likely an underestimate due to observational problems. ${ }^{\dagger}$ NGC 2329 shows possible signs of inflow.

We were able to correlate observed and modeled kinematic patterns in 12 of the 21 galaxies in the sample and assign them ranges of black hole masses, which we list in Table 2. For the remainder of the galaxies the point to point variations in the velocity profiles are greater than the differences between the varying mass models. In NGC 2329 we observe a shallow velocity profile indicating that flow (non-circular inward or outward motions) may be important; supported by inward and outward motions observed in the side slits along the minor axis. The softening of the rotation curves by this type of flow may also be significant, though harder to detect in other nuclei, and could have implications for black hole masses derived by modeling gas disks.

\section{Acknowledgements}

Support for this work was provided in part by grants HST GO-08236.02, HST GO09495-07A and SAO GO3-4139B. J.N.-S. thanks Isaac Gerstein and family for their hospitality while completing this paper. The authors thank R. van der Marel, J. van Gorkom and P. T. de Zeeuw for many useful conversations on the topics presented here.

\section{References}

Barth, A. J., Filippenko, A. V., \& Moran, E. C. 1999, ApJ, 525

Brown, T., et al. 2002, in HST STIS Data Handbook, 4th edn., ed. B. Mobasher (Baltimore, MD: STScI)

Fanaroff, B. L., \& Riley, J. M. 1974, MNRAS, 167, 31P

Ho, L. C., Filippenko, A. V., Sargent, W. L. W., \& Peng, C. Y. 1997, ApJS, 112, 391

Nilson, P. 1973, The Uppsala General Catalogue of Galaxies [UGC] (Uppsala Observatory)

Noel-Storr, J. 2004, PhD thesis, Columbia University, New York, NY

Noel-Storr, J., et al. 2003, ApJS, 148, 419

Sulentic, J. W., Marziani, P., \& Dultzin-Hacyan, D. 2000, ARA\&A, 38, 521

Verdoes Kleijn, G. A., Baum, S. A., de Zeeuw, P. T., \& O’Dea, C. P. 1999, AJ, 118, 2592

Xu, C., Baum, S. A., O'Dea, C. P., Wrobel, J. M., \& Condon, J. J. 2000, AJ, 120, 2950 